\title{
Characterizing Storytelling in COVID-19 Cartographic Journalism
}

\author{
Timothy Prestby ${ }^{\mathrm{a}}$ \\ ${ }^{a}$ The Pennsylvania State University, GeoVISTA Center (USA), tprestby@psu.edu
}

Keywords: visual storytelling, data journalism, story maps, cartographic design, health communication

\section{Problem}

The SARS-CoV-2 virus (COVID-19) first appeared in November 2019 in Wuhan, China. In March 2020, the virus evolved from an epidemic into a pandemic as large populations in Europe, South, and North America became infected. Maps covering topics ranging from cases to deaths to social and economic effects of the COVID-19 pandemic have simultaneously exploded as the world struggles to come to grips with an unfamiliar, scary reality. Mapmakers hold more responsibility and power than ever as their design choices and products help shape the collective consciousness of epidemiologists, policy makers, and the public at large. While there is abundant research on mapping disease and healthrelated concepts for scientific analysis, research investigating the role of map design in augmenting health communication remains scarce. This work builds on this area of deficit by examining aspects of these maps and the overall story map that may engage, persuade, and change behaviour of readers. My work seeks to identify common design strategies of COVID-19 story maps in journalism to guide future map-based health communication and human-subject experiments that evaluate the effectiveness of identified design techniques.

\section{Background}

Public health depends on clear and tangible communication. Health communication may be particularly effective if structured as a sequential story that has some variation of a beginning, middle and end known as a narrative [1]. To understand the expanding role of narratives in health communication we must evaluate the effectiveness of different narrative mediums. Maps are one such medium. While maps have always been rooted in narratives and storytelling, only recently have cartography and related fields focused significant attention and room in their research agendas to the intersections of maps and stories [2]. Stories that not only include a map, but are driven by the map and other visuals are defined as story maps. Story maps are popular to the public thanks to their capacity to fuse a wide range of multimedia together, present on a multitude of devices, afford interactivity, and personalize the experience (Roth, 2020). Another driver of story maps has come from data journalists pushing the boundaries of narrative visualizations based on the improvements in technology and data availability (Seyser \& Zeiller, 2018).

Story maps come in many forms. However, every story map follows a narrative that glues the content of the plot together to produce the overall story (Roth, 2020). What separates story maps from one another is their structure in terms of genre and tropes. Genre refers to how linearity is enforced in the narrative, whereas tropes refer to literary devices that advance the story while enriching the narrative (Roth, 2020). Tropes come to life through an eclectic set of visual design techniques that are implemented throughout the narrative and its elements rather than the visualized data. While Roth identifies seven categories of genres and tropes, we do not know how these genres and tropes are utilized in cartographic media.

Research on evaluating disease mapping as a communication medium remain largely untapped. Maps may be particularly effective as a communication tool as they are a familiar and intuitive way of presenting spatial information to a wide spectrum of people. Bell et al. [3] underscores the importance of producing health-related maps that follow wellestablished cartographic design principles.

Simply following cartographic design principles may not be enough to for a map to become persuasive. A map needs to draw readers in and sustain their interest by stimulating their imagination and appealing to their emotions, and it is theorized to do this through its vividness [4]. Vividness refers to content that appeals to readers emotions and makes content relatable and alive, so they are more likely to become immersed in the narrative. Thus, vividness acts as a persuasive mechanism through which readers may become open to absorb information that contradicts their worldview. Although vividness can influence the attitudes of readers, we need to know more about the underlying persuasive design mechanisms of vivid content in maps.

The conceptualization of vividness has only recently been taken up in cartographic research. Fish [5] uses vividness as a lens to evaluate the power of climate change maps in visual communication. In previous work, other cartographic scholars 
have studied aspects of vividness including emotion, salience, persuasion, and intimacy. Maps are a particularly persuasive visual as people tend to trust what they represent more so than if the same information another form like text [6]. Therefore, understanding how to leverage vividness in mapmaking is paramount as the public may be easily influenced by them for good or for bad.

\section{Methods}

I implemented a quantitative content analysis building on the approach outlined by Muehlenhaus (2011) to evaluate which aspects of COVID-19 the story maps cover, what design traits of vividness they used, and to identify which visual storytelling techniques they employ. Because my goal is to characterize design traits related to vividness and storytelling elements, I apply content analysis using a coding scheme that combines Roth's [2] visual storytelling and Fish's [7] vividness frameworks.

The sample consisted of 182 COVID-19 story maps published between January and December 2020 that communicate the arc of the pandemic up to the start of vaccination campaigns. These maps were collected via keyword search in social media sources. A list of Twitter accounts was created to organize the works of major newsroom graphics teams in one place. This list includes Twitter presences associated with the data, visuals, or graphics teams. Since not all newsrooms have Twitter presences, the same keyword searches were applied in Google searches to find additional media outlets. The final set featured 21 newsrooms including 1) The Washington Post, 2) Reuters, 3) The Times, 4) South China Morning Post, 5) Canadian Broadcasting Corporation, and 6) ABC News Australia. Although the resulting purposeful sample of map stories is not exhaustive, it represents a broad swath of typical works from mainstream newsrooms based in the United States. Figure 1 presents a segment of a story map constructed as the genre longform infographic and shows the tropes attention, metaphor, and the vividness element visual salience.

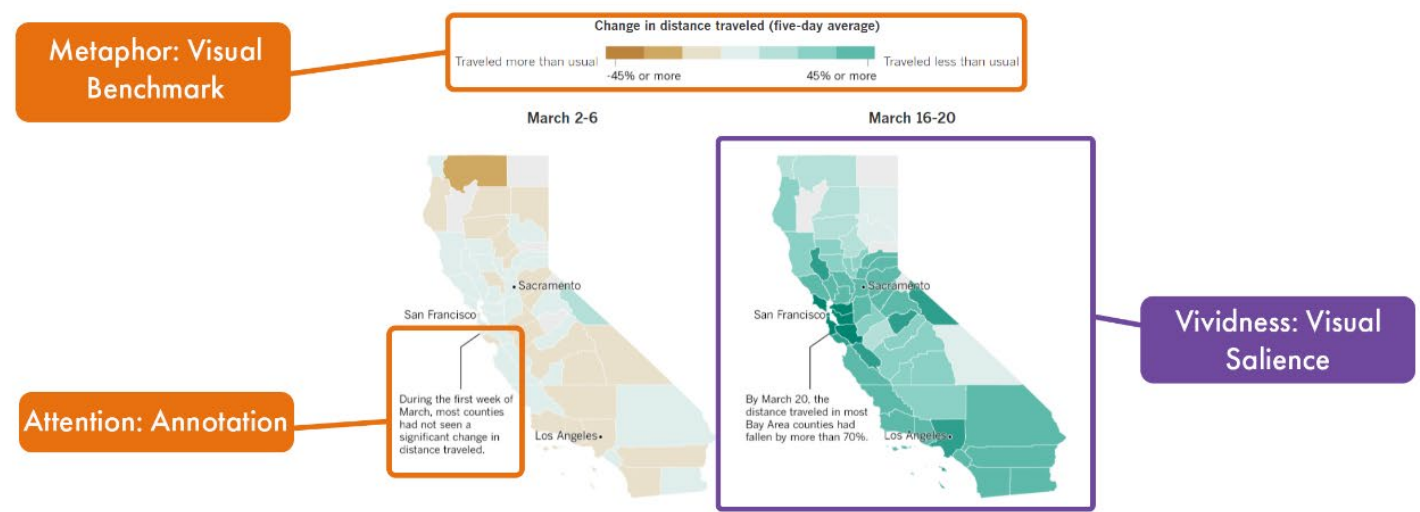

Figure 1. Example of Coding Scheme done on "Empty freeways, cancelled flights: How Life in California has changed since the coronavirus outbreak" by The Los Angeles Times

Outlook: The next steps of this work will involve coding the map collection against the combined storytelling and vividness frameworks. I anticipate having the raw data later in 2021, so that statistical analysis can be performed. I plan to perform simple descriptive statistics to calculate the frequencies of storytelling tropes and attributes of vividness. I also intend to perform cross-tabulations to test for relationships between tropes and vividness variables.

\section{References:}

[1] L. J. Hinyard and M. W. Kreuter, "Using Narrative Communication as a Tool for Health Behavior Change: A Conceptual, Theoretical, and Empirical Overview," Health Educ Behav, vol. 34, no. 5, pp. 777-792, Oct. 2007, doi: 10.1177/1090198106291963.

[2] R. E. Roth, "Cartographic Design as Visual Storytelling: Synthesis and Review of Map-Based Narratives, Genres, and Tropes," null, pp. 1-32, Sep. 2020, doi: 10.1080/00087041.2019.1633103.

[3] B. S. Bell, R. E. Hoskins, L. W. Pickle, and D. Wartenberg, "Current practices in spatial analysis of cancer data: mapping health statistics to inform policymakers and the public," International Journal of Health Geographics, vol. 5, no. 1, p. 49, Nov. 2006, doi: 10.1186/1476-072X-5-49.

[4] R. E. Nisbett and L. Ross, Human inference: Strategies and shortcomings of social judgment. Prentice-Hall, 1980.

[5] C. Fish, "Cartographic content analysis of compelling climate change communication," Cartography and Geographic Information Science, vol. 47, no. 6, pp. 492-507, Nov. 2020, doi: 10.1080/15230406.2020.1774421.

[6] J. A. Tyner, "Persuasive cartography," Journal of Geography, vol. 81, no. 4, pp. 140-144, 1982.

[7] C. Fish, "Elements of Vivid Cartography," The Cartographic Journal, vol. 0, no. 0, pp. 1-17, Jan. 2021, doi: 10.1080/00087041.2020.1800160. 